\title{
Analytical method for perturbed frozen orbit around an Asteroid in highly inhomogeneous gravitational fields: a first approach.
}

\author{
Marta Ceccaroni Francesco Biscani James Biggs
}

\begin{abstract}
This article provides a method for finding initial conditions for perturbed frozen orbits around inhomogeneous fast rotating asteroids. These orbits can be used as reference trajectories in missions that require close inspection of any rigid body. The generalized perturbative procedure followed exploits the analytical methods of relegation of the argument of node and Delaunay normalisation to arbitrary order. These analytical methods are extremely powerful but highly computational.

The gravitational potential of the heterogeneous body is firstly stated, in polar-nodal coordinates, which takes into account the coefficients of the spherical harmonics up to an arbitrary order. Through the relegation of the argument of node and the Delaunay normalization, a series of canonical transformations of coordinates is found, which reduces the Hamiltonian describing the system to a integrable, two degrees of freedom Hamiltonian plus a truncated reminder of higher order. Setting eccentricity, argument of pericenter and inclination of the orbit of the truncated system to be constant, initial conditions are found, which evolve into frozen orbits for the truncated system. Using the same initial conditions yields perturbed frozen orbits for the full system, whose perturbation decreases with the consideration of arbitrary homologic equations in the relegation and normalization procedures. Such procedure can be automated for the first homologic equation up to the consideration of any arbitrary number of spherical harmonics coefficients. The project has been developed in collaboration with the European Space Agency (ESA).
\end{abstract}

Marta Ceccaroni

Advanced Space Concepts Laboratory University of Strathclyde, Glasgow, UK

marta.ceccaroni@strath.ac.uk

Francesco Biscani

Advanced Concepts Team

ESTEC: European Space Research and Technology Centre

Francesco.Biscani@esa.int

James Biggs

Advanced Space Concepts Laboratory

University of Strathclyde, Glasgow, UK

james.biggs@strath.ac.uk 


\section{Introduction}

The motion of bodies subject to non-Keplerian gravitational fields is a classical subject of research in the context of celestial mechanics. In recent years this type of research has become important to future planned missions of spacecraft to the moon and asteroids in addition to asteroid deflection missions such as the European Space Agency's "Don Quijote" concept Carnelli and Gálvez (2006). Research undertaken in this area has studied the effect of the Earth's inhomogeneous gravitational field on the motion of natural and artificial satellites, that is, artificial satellite theory for small and moderate eccentricities Deprit (1970). More recent studies have researched the effects on motion of the inhomogeneous gravitational field of other solar system bodies, including the Moon Abad et al. (2009) and asteroids San-Juan et al. (2004). The analysis of spacecraft motion about these bodies is particularly challenging as they typically feature shapes and density distributions more irregular than those of planets. Such irregularities break symmetries and require more complicated analytical expressions for their description which increases the complexity involved in such studies.

Numerical methods are today widely used to study the trajectories of objects orbiting specific irregular bodies ( see Fahnestock and Scheeres (2008) or Colombo et al. (2012)) or for finding stability criteria (Lara and Scheeres (2002)). Disadvantages of these methods are that they can be highly computational and require a complete re-design for each different body. Analytical methods, by contrast, have the potential to rapidly identify useful natural motions for general bodies with inhomogeneous gravitational fields. Furthermore, they can provide a full dynamical picture of the motion around irregular bodies that can be used to search and study particular classes of useful orbits. However, current analytical methods are only used in a limited and semi-numerical way (meaning that analytical expansions constitute the first step in such studies, which are then typically carried out from a numerical standpoint Scheeres et al. (1998)). The main drawbacks of these methods are that their application in the case of highly inhomogeneous bodies requires extensive symbolic computations involving algebraic manipulations, and that they are usually restricted to a certain range of eccentricities due to series convergence. Analytical studies on inhomogeneous gravitational fields have been, so far, limited to low degree gravity fields Palacian (2002), San-Juan et al. (2002), San-Juan et al. (2004), thus restricting the results to a class of bodies for which the dynamics is dominated by a few coefficients (e.g. oblateness or ellipticity).

In this paper a closed form (i.e. without using series expansion in the eccentricity), analytical, perturbative theory of motion around inhomogeneous bodies is presented, generalized to second order, arbitrary degree gravity fields. Using Deprit's method (Deprit (1969)) to construct two different Lie transformations suitable canonical action-angle variables are found, which reduce the initial nonintegrable Hamiltonian into an integrable one plus a negligible, perturbative remainder.

The method can be used to find useful orbits for space mission applications such as frozen orbits. Frozen orbits are orbits with no secular perturbations in the inclination, argument of pericenter, and eccentricity (Brouwer (1959)). These orbits are periodic orbits, except for the orbital plane of precession, and are therefore called frozen. 


\section{Method}

Assuming that the planetary body is in uniform rotation around its axis of greatest inertia, the potential generated by the inhomogeneous gravitational field can be derived in the rotating polar nodal variables (Whittaker (1917)) convenient for the necessary transformation to Delaunay coordinates. This potential takes into account an arbitrary number of spherical harmonic coefficients, all considered to have the same order, thus providing a dynamical model based on an arbitrarily accurate model of the inhomogeneous body. For the case of fast rotating asteroids, where the Coriolis term is bigger than the two body potential, the methodology is then based on the following steps:

- Relegation of the argument of node to obtain the relegated nodal variables where the longitude of nodes conjugate momenta is constant along the Hamiltonian flow.

- Transformation to Delaunay variables to yield a constant total angular momentum in the z-direction

- Normalization of the Delaunay variables which yields a reduced ordinary differential equation in two coordinates; the total angular momentum and the argument of pericentre

- Frozen orbits identified as the equilibrium points of these equations i.e. where the total angular momentum about the z-axis and the argument of pericentre are constant, therefore the final stage is undertaken by solving a $2-\mathrm{D}$ algebraic equation.

The methodology comprises of two different Lie transformations, relegation and normalisation, constructed following Deprit's method for canonic transformations (Deprit (1969)). The usual technique applied previously in the literature i.e. the Delaunay normalization (Deprit (1982)), cannot be directly applied to a high-order model due to the presence of the longitude of nodes that appears in the Coriolis term. The addition of this term in the Lie derivative prevents the conventional computation of the Lie transform generator (San-Juan et al. (2002)). However, Palacián's closed form relegation algorithm (Palacián (1992)) can be applied, which "relegates" the action of the longitude of nodes to a negligible remainder. It is shown that, for this model, both relegation and normalization results are equivalent to averaging over the fast angles.

\section{The dynamical system}

An inhomogeneous body is considered, which rotates uniformly around its axis of greatest inertia with constant angular velocity $\hat{\omega}=[0,0, \omega]$.

The total mass of the body is $M$ while $\mathcal{G}$ is the universal gravitational constant. The dynamics is formulated into a reference frame centered in the center of mass of the body and oriented with the "z-axis" parallel to the rotational axis of the asteroid. The frame of reference is rotating with the same velocity of rotation of the asteroid; in such rotating coordinates the Hamiltonian describing the system is:

$$
H(\mathbf{x}, \mathbf{X})=\frac{1}{2}(\mathbf{X} \cdot \mathbf{X})-\hat{\omega}(\mathbf{x} \times \mathbf{X})+\bar{U}(\mathbf{x})
$$


where $\mathbf{x}, \mathbf{X} \in \mathbb{R}^{3}$ are respectively the position coordinates and conjugate momenta of the spacecraft, while $\bar{U}(\mathbf{x})$ is the perturbing gravitational potential generated by the inhomogeneous rotating body. The equations of motion are:

$$
\left\{\begin{array}{l}
\dot{\mathbf{x}}=\frac{\partial}{\partial \mathbf{X}} H(\mathbf{x}, \mathbf{X}) \\
\dot{\mathbf{X}}=-\frac{\partial}{\partial \mathbf{x}} H(\mathbf{x}, \mathbf{X})
\end{array}\right.
$$

It is convenient to express the Hamiltonian and the perturbing potential using the so called nodal-polar variables so that it may easily be transformed to the Delaunay coordinates in the later stage of the methodology: $r=|\mathbf{x}|, \theta$ is the argument of latitude, and $\nu$ the longitude of the ascending node and their respective conjugate momenta $R, \Theta$, and $N$. The transformation required is given in Palacian (2002), setting $\mathbf{x}=[x, y, z]^{T}$ and $\mathbf{X}=[X, Y, Z]^{T}$ :

$$
\begin{aligned}
& x=r(\cos \theta \cos \nu-\sin \theta \cos I \sin \nu) \\
& y=r(\cos \theta \sin \nu+\sin \theta \cos I \cos \nu) \\
& z=r \sin \theta \sin I \\
& X=\left(R \cos \theta-\frac{\Theta}{\mid \mathbf{r}} \sin \theta\right) \cos \nu-\left(R \sin \theta+\frac{\Theta}{|\mathbf{r}|} \cos \theta\right) \cos I \sin \nu \\
& Y=\left(R \cos \theta-\frac{\Theta}{|\mathbf{r}|} \sin \theta\right) \sin \nu+\left(R \sin \theta+\frac{\Theta}{|\mathbf{r}|} \cos \theta\right) \cos I \cos \nu \\
& Z=\left(R \sin \theta+\frac{\Theta}{|\mathbf{r}|} \cos \theta\right) \sin I
\end{aligned}
$$

In these coordinates the Hamiltonian takes the form:

$$
H(r, \theta, \nu, R, \Theta, N)=\frac{1}{2}\left(R^{2}+\frac{\Theta^{2}}{r^{2}}\right)-\omega N+\bar{U}(r, \theta, \nu, R, \Theta, N)
$$

where $N=\Theta \cos I$. The expression for the gravitational potential is derived as follows.

\section{The gravitational potential}

Consider an arbitrarily shaped body $B$ of finite extension; denote with $\mathbf{r}^{\prime} \in$ $\mathbb{R}^{3}$ the position of the infinitesimal mass element $d M$ in a cartesian reference frame $O_{x y z}$. The gravity potential of such a continuous mass distribution on an

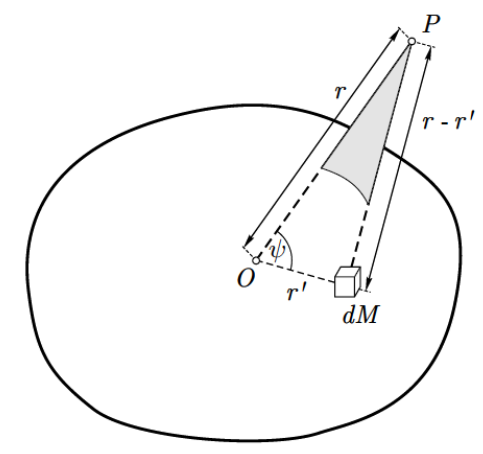

Figure 1: The potential generated by an arbitrarily shaped body $B$ is the integral over the volume the infinitesimal mass elements $d M$ 
external point $P$ set in $\mathbf{r} \in \mathbb{R}^{3}$ is:

$$
U(\mathbf{r})=-\mathcal{G} \int_{V} \frac{\rho\left(\mathbf{r}^{\prime}\right)}{\left|\mathbf{r}-\mathbf{r}^{\prime}\right|} d V
$$

where $\rho\left(\mathbf{r}^{\prime}\right)$ is the density of the body and $d V$ is the infinitesimal element of volume (i.e. $d M=\rho\left(\mathbf{r}^{\prime}\right) d V$ ) and $V$ is the volume of the body.

As it is well known:

$$
U(r)=-\frac{\mathcal{G}}{r} \int_{V} \sum_{n=0}^{\infty}\left(\frac{r^{\prime}}{r}\right)^{n} P_{n}(\cos (\psi)) \rho\left(\mathbf{r}^{\prime}\right) d V .
$$

Here $r=|\mathbf{r}|, r^{\prime}=\left|\mathbf{r}^{\prime}\right|$.

The condition $\frac{r^{\prime}}{r}<1$ implies that the model is valid only outside the reference sphere that is conventionally taken as the sphere circumscribing the asteroid.

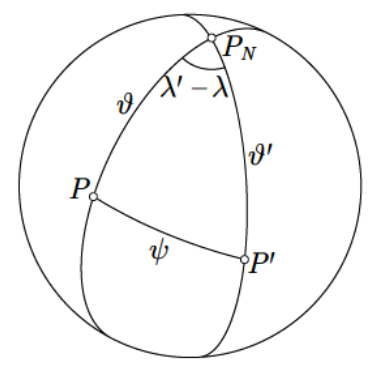

Figure 2: The angle $\psi$ can be expressed in terms of the latitude $\delta$ and longitude $\lambda$

To get the expression of the gravitational potential into the Wittaker NodalPolar variables, the non scaled spherical harmonics and Wigner's rotation theorem will be used.

Definition 1 The non scaled spherical harmonics $Y_{n}^{m}(\delta, \lambda)$ are the angular portion of the solution to Laplace's equation in spherical coordinates where azimuthal symmetry is not present, which can be expressed as

$$
Y_{n}^{m}(\delta, \lambda):=P_{n}^{m}(\sin \delta) e^{\imath m \lambda}
$$

The addition formula for non scaled spherical harmonics Hofmann-Wellenhof et al. (1967) it therefore applied to (6):

$$
P_{n}(\cos \psi)=\Re\left[\sum_{m=0}^{n}(-1)^{m}\left(2-\delta_{0, m}\right) Y_{n}^{-m}(\delta, \lambda) Y_{n}^{m}\left(\delta^{\prime}, \lambda^{\prime}\right)\right]
$$


Thus obtaining:

$$
\begin{aligned}
U(r, \delta, \lambda))=-\frac{\mathcal{G}}{r} \int_{V} \sum_{n=0}^{\infty}\left(\frac{r^{\prime}}{r}\right)^{n} \Re\left[\sum_{m=0}^{n}(-1)^{m}\left(2-\delta_{0, m}\right) Y_{n}^{-m}(\delta, \lambda) Y_{n}^{m}\left(\delta^{\prime}, \lambda^{\prime}\right)\right] . \\
\cdot \rho\left(\mathbf{r}^{\prime}\right) d V \\
=-\frac{\mathcal{G} M}{r} \sum_{n=0}^{\infty} \Re\left[\sum _ { m = 0 } ^ { n } ( - 1 ) ^ { m } ( \frac { \alpha } { r } ) ^ { n } Y _ { n } ^ { - m } ( \delta , \lambda ) \left(\frac{1}{M} \int_{V}\left(\frac{r^{\prime}}{\alpha}\right)^{n}\left(2-\delta_{0, m}\right) .\right.\right. \\
\left.\left.\cdot Y_{n}^{m}\left(\delta^{\prime}, \lambda^{\prime}\right) \rho\left(\mathbf{r}^{\prime}\right) d V\right)\right] \\
=\left[-\frac{\mathcal{G} M}{r} \sum_{n=0}^{\infty} \sum_{m=0}^{n}\left(\frac{\alpha}{r}\right)^{n} \frac{(n+m) !}{(n-m) !} Y_{n}^{-m}(\delta, \lambda)\left(\frac{1}{M} \int_{V}\left(\frac{r^{\prime}}{\alpha}\right)^{n}\left(2-\delta_{0, m}\right) .\right.\right. \\
\left.\left.\cdot \frac{(n-m) !}{(n+m) !} P_{n, m}\left(\sin \delta^{\prime}\right) e^{\imath m \lambda^{\prime}} \rho\left(\mathbf{r}^{\prime}\right) d V\right)\right] \\
\qquad\left[-\frac{\mathcal{G} M}{r} \sum_{n=0}^{\infty} \sum_{m=0}^{n}\left(\frac{\alpha}{r}\right)^{n} \frac{(n+m) !}{(n-m) !} Y_{n}^{-m}(\delta, \lambda) K_{n, m}\right]
\end{aligned}
$$

where $K_{n, m}=C_{n, m}+\imath S_{n, m}$ and $C_{n, m}$ and $S_{n, m}$ are called spherical harmonic coefficients or "Stokes coefficient", defined as, $\forall 0 \leq m \leq n$ :

$$
\begin{aligned}
C_{n, m} & =\frac{\left(2-\delta_{m, 0}\right)}{M} \frac{(n-m) !}{(n+m) !} \int_{V}\left(\frac{r^{\prime}}{\alpha}\right)^{n} P_{n, m}\left(\sin \delta^{\prime}\right) \cos \left(m \lambda^{\prime}\right) \rho\left(r^{\prime}, \delta^{\prime}, \lambda^{\prime}\right) d V \\
S_{n, m} & =\frac{2}{M} \frac{(n-m) !}{(n+m) !} \int_{V}\left(\frac{r^{\prime}}{\alpha}\right)^{n} P_{n, m}\left(\sin \delta^{\prime}\right) \sin \left(m \lambda^{\prime}\right) \rho\left(r^{\prime}, \delta^{\prime}, \lambda^{\prime}\right) d V
\end{aligned}
$$

where $\alpha$ is the radius of the reference sphere of the small body.

Moreover $\delta_{0, m}$ is the Kronecker delta that gives 1 if $m=0$, and 0 elsewhere, $P_{n}^{m}(x)$ is the associated Legendre function of degree $n$ and order $m$.

Also $\mathbf{r}^{\prime} \in \mathbb{R}^{3}, \theta^{\prime} \in \mathbb{R}$ and $\lambda^{\prime} \in \mathbb{R}$ are respectively the position, latitude and longitude of the infinitesimal volume element $d V$ in a cartesian frame of reference $O_{x, y, z}$.

A full explanation of the spherical harmonic coefficients can be found in Hofmann-Wellenhof et al. (1967).

However it is important to highlight that, in particular, the equations in (10) imply that:

$$
\begin{aligned}
& C_{0,0}=1 \\
& C_{n, 0}=\frac{1}{M} \int_{V}\left(\frac{r^{\prime}}{\alpha}\right)^{n} P_{n}\left(\sin \delta^{\prime}\right) \rho\left(r^{\prime}, \delta^{\prime}, \lambda^{\prime}\right) d V \quad \forall n>0 \\
& S_{n, 0}=0 \quad \forall n \geq 0
\end{aligned}
$$

Moreover, centering the origin of the system of reference at the center of mass it can be demonstrated that the term $C_{1,0}=C_{1,1}=S_{1,1}=0$. 
The coefficients $C_{2,0}$ and $C_{2,2}$ express the "ellipticity" and "oblateness" of the body.

Now we apply Wigner's rotation theorem for non scaled spherical harmonics (see Wigner (1959)) in order to get to the nodal polar variables.

Theorem $1 \forall n, m \in \mathbb{N}, n, m$ let be $Y_{n}^{m}(\delta, \lambda)$ the spherical harmonics expressed in terms of the latitude $\delta$ and longitude $\lambda$ in a system of reference $O_{\hat{\boldsymbol{x}}, \hat{\mathbf{y}}, \hat{\mathbf{z}}}$. Then the expression for $Y_{n}^{m}(\delta, \lambda)$ in terms of the latitude $\Delta$ and longitude $\Lambda$ in another system of reference $O_{x, y, z}$, obtained by the composition of three rotations of angles $\alpha, \beta$ and $\gamma$ (around the $\hat{\boldsymbol{x}}$ axis, the rotated $\hat{\boldsymbol{z}}$, and the rotated $\hat{\boldsymbol{x}}$ axis respectively), is given by:

$$
Y_{n}^{m}(\delta, \lambda)=\sum_{j=-n}^{n} D_{j, m}^{n}(-\alpha,-\beta,-\gamma) Y_{n}^{j}(\Delta, \Lambda)
$$

where

$$
D_{j, m}^{n}(-\alpha,-\beta,-\gamma)=e^{\imath j\left(\alpha+\frac{\pi}{2}\right)} e^{\imath m\left(\gamma-\frac{\pi}{2}\right)} d_{j, m}^{n}(-\beta)
$$

and

$$
\begin{aligned}
& d_{j, m}^{n}(-\beta)=\sum_{t=\max \{0, j-m\}}^{\min \{n-m, n+j\}}(-1)^{m-j+3 t} \frac{(n-j) !(n+m) !}{t !(n+j-t) !(n-m-t) !(m-j+t) !} . \\
& \cdot\left(\cos \left(\frac{\beta}{2}\right)\right)^{2 n-(m-j+2 t)}\left(\sin \left(\frac{\beta}{2}\right)\right)^{m-j+2 t}
\end{aligned}
$$

We want to apply this theorem by setting the second system of reference to be the one where the spacecraft position vector is $(0,0, r)$ therefore the three angles $\alpha, \beta$ and $\gamma$ are set to be $\theta, I$ and $\nu$, the argument of latitude, the inclination of the orbital plane and namely the longitude of the ascending node; moreover it must be noticed that in such system of reference the new latitude $\Delta$ and longitude $\Lambda$ of the spacecraft will be both equal to zero as we have set its new 
position vector to be $(0,0, r)$. Therefore $(9)$ becomes:

$$
\begin{aligned}
& U(r, \delta, \lambda))=\Re\left[-\frac{\mathcal{G} M}{r} \sum_{n=0}^{\infty} \sum_{m=0}^{n}\left(\frac{\alpha}{r}\right)^{n} \frac{(n+m) !}{(n-m) !} \sum_{j=-n}^{n} D_{j,-m}^{n}(-\alpha,-\beta,-\gamma)\right. \\
& \left.Y_{n}^{j}(0,0) K_{n, m}\right] \\
& =\Re\left[-\frac{\mathcal{G} M}{r} \sum_{n=0}^{\infty} \sum_{m=0}^{n}\left(\frac{\alpha}{r}\right)^{n} \frac{(n+m) !}{(n-m) !} \sum_{j=-n}^{n} D_{j,-m}^{n}(-\theta,-I,-\nu) Y_{n}^{j}(0,0) K_{n, m}\right] \\
& =\Re\left[-\frac{\mathcal{G} M}{r} \sum_{n=0}^{\infty} \sum_{m=0}^{n}\left(\frac{\alpha}{r}\right)^{n} \frac{(n+m) !}{(n-m) !} \sum_{j=-n}^{n} e^{\imath(j \theta-m \nu)} e^{\imath \frac{\pi}{2}(k+m)} d_{j,-m}^{n}(-I) .\right. \\
& \left.\cdot P_{n}^{j}(0) K_{n, m}\right] \\
& =-\frac{\mathcal{G} M}{r} \sum_{n=0}^{\infty} \sum_{m=0}^{n}\left(\frac{\alpha}{r}\right)^{n} \frac{(n+m) !}{(n-m) !} \sum_{j=-n}^{n} \sum_{\ell=\max \{0, j+m\}}^{\min \{n+m, n+j\}}(-1)^{m-j+3 \ell} . \\
& \cdot \frac{(n-j) !(n-m) !}{\ell !(n+j-\ell) !(n+m-\ell) !(-m-j+\ell) !}\left(\cos \left(\frac{I}{2}\right)\right)^{2 n-(-m-j+2 \ell)}\left(\sin \left(\frac{I}{2}\right)\right)^{-m-j+2 \ell} . \\
& \cdot\left((-1)^{j}\left((n+j)_{\equiv_{2}}-1\right)(-1)^{\frac{n-j}{2}} \frac{(n+j-1) ! !}{2^{\frac{n-j}{2}}\left(\frac{n-j}{2}\right) !}\right) \cdot \\
& \cdot\left(C_{n, m}\left(\cos (j \theta-m \nu) \cos \left(\frac{\pi}{2}(j+m)\right)-\sin (j \theta-m \nu) \sin \left(\frac{\pi}{2}(j+m)\right)\right)\right. \\
& \left.+S_{n, m}\left(-\sin (j \theta-m \nu) \cos \left(\frac{\pi}{2}(j+m)\right)-\cos (j \theta-m \nu) \sin \left(\frac{\pi}{2}(j+m)\right)\right)\right) \\
& =-\frac{\mathcal{G} M}{r} \sum_{n=0}^{\infty} \sum_{m=0}^{n}\left(\frac{\alpha}{r}\right)^{n} \sum_{j=-n}^{n} \sum_{\ell=\max \{0, j+m\}}^{\min \{n+m, n+j\}}(-1)^{m-j+3 \ell} \text {. } \\
& \cdot \frac{(n-j) !(n+m) !}{\ell !(n+j-\ell) !(n+m-\ell) !(-m-j+\ell) !}\left(\cos \left(\frac{I}{2}\right)\right)^{2 n-(-m-j+2 \ell)}\left(\sin \left(\frac{I}{2}\right)\right)^{-m-j+2 \ell} . \\
& \cdot(-1)^{\frac{n+j}{2}} \frac{1}{2^{n}} \frac{(n+j) !}{\left(\frac{n+j}{2}\right) !\left(\frac{n-j}{2}\right) !}\left((n+j)_{\equiv_{2}}-1\right) \frac{(n+m) !}{2^{n}\left(\frac{n-m}{2}\right) !\left(\frac{n+m}{2}\right) !} . \\
& \cdot\left(C_{n, m}\left(\cos (j \theta-m \nu) \cos \left(\frac{\pi}{2}(j+m)\right)-\sin (j \theta-m \nu) \sin \left(\frac{\pi}{2}(j+m)\right)\right)\right. \\
& \left.+S_{n, m}\left(-\sin (j \theta-m \nu) \cos \left(\frac{\pi}{2}(j+m)\right)-\cos (j \theta-m \nu) \sin \left(\frac{\pi}{2}(j+m)\right)\right)\right)
\end{aligned}
$$


calling

$$
\begin{aligned}
\overline{\mathcal{G}}_{n, m, j}(I) & =\sum_{\ell=\max \{0, j+m\}}^{\min \{n+m, n+j\}}(-1)^{m+3 \ell-j} \frac{(n+m) !(n-j) !}{\ell !(n+j-\ell) !(n+m-\ell) !(\ell-m-j)} . \\
\cdot(-1)^{\frac{n+j}{2}} & \frac{1}{2^{n}} \frac{(n+j) !}{\left(\frac{n+j}{2}\right) !\left(\frac{n-j}{2}\right) !}\left((n+j)_{\equiv_{2}}-1\right) \cos \left(\frac{I}{2}\right)^{2 n+m+j-2 \ell} \sin \left(\frac{I}{2}\right)^{2 \ell-m-j}
\end{aligned}
$$

where $x_{\equiv_{y}}$ stands for the value of $x$ modulus $y$, i.e. the integer reminder of the division of $x$ by $y$.

The potential can be rearranged as:

$$
\begin{aligned}
& U(r, \theta, \nu)=-\frac{\mathcal{G} M}{r} \sum_{n=0}^{\infty}\left(\frac{\alpha}{r}\right)^{n} \sum_{m=0}^{n} \sum_{j=-n}^{n} \overline{\mathcal{G}}_{n, m, j}(I) . \\
& \quad \cdot\left(C_{n, m}\left(\cos (j \theta-m \nu) \cos \left(\frac{\pi}{2}(j+m)\right)-\sin (j \theta-m \nu) \sin \left(\frac{\pi}{2}(j+m)\right)\right)+\right. \\
& \left.\quad+S_{n, m}\left(-\sin (j \theta-m \nu) \cos \left(\frac{\pi}{2}(j+m)\right)-\cos (j \theta-m \nu) \sin \left(\frac{\pi}{2}(j+m)\right)\right)\right)
\end{aligned}
$$

Finally we rearrange the potential in a way that will be useful to apply the relegation algorithm described in the next section:

$$
\begin{aligned}
& U(r, \theta, \nu)=-\frac{\mathcal{G} M}{r} \sum_{n=0}^{\infty}\left(\frac{\alpha}{r}\right)^{n} \sum_{m=0}^{n} \sum_{j=-n}^{n}\left(\mathcal{G}_{n, m, j}^{1}(I) \cos (m \nu-j \theta)+\mathcal{G}_{n, m, j}^{2}(I) .\right. \\
& \cdot \sin (m \nu-j \theta)),
\end{aligned}
$$

with:

$$
\begin{aligned}
& \mathcal{G}_{n, m, j}^{1}(I)=\overline{\mathcal{G}}_{n, m, j}(I)\left(C_{n, m} \cos \left(\frac{\pi}{2}(j+m)\right)-S_{n, m} \sin \left(\frac{\pi}{2}(j+m)\right)\right) \\
& \mathcal{G}_{n, m, j}^{2}(I)=\overline{\mathcal{G}}_{n, m, j}(I)\left(C_{n, m} \sin \left(\frac{\pi}{2}(j+m)\right)+S_{n, m} \cos \left(\frac{\pi}{2}(j+m)\right)\right),
\end{aligned}
$$

and $\overline{\mathcal{G}}_{n, m, j}(I)$ as in (16). Again, recall that series (18) converges, which implies that the model is valid only outside the reference sphere.

\section{$5 \quad$ Lie Transformations}

A short description of Deprit's method for Lie Transformations, with respect to our application, is here provided, while for a full description of it and a comparison with the Von Zeipel's method which determines the Lie transformations we refer the reader to Deprit (1969).

Definition $2 A$ Lie transformation $\phi$ is a one-parameter family of mappings $\phi:(y, Y ; \epsilon) \rightarrow(x, X)$, defined by the solution $x(y, Y ; \epsilon)$ and $X(y, Y ; \epsilon)$ of the Hamiltonian system

$$
\left\{\begin{array}{l}
\frac{d x}{d \epsilon}=\frac{\partial W}{\partial X} \\
\frac{d X}{d \epsilon}=-\frac{\partial W}{\partial x}
\end{array}\right.
$$


With initial conditions $x(y, Y ; 0)=y$ and $X(y, Y ; 0)=Y$, and where the function

$$
W(x, X ; \epsilon)=\sum_{s \geq 0} \frac{\epsilon^{s}}{s !} W_{s+1}(x, X)
$$

is the generator of the transformation.

Due to the properties of the Hamiltonian systems, the Lie transformation $\phi$ is a completely canonical transformation that maps a Hamiltonian

$$
H(x, X ; \epsilon)=\sum_{s \geq 0} \frac{\epsilon^{s}}{s !} H_{s}(x, X)
$$

onto an equivalent Hamiltonian $K$ of the form

$$
K(y, Y ; \epsilon)=\sum_{s \geq 0} \frac{\epsilon^{s}}{s !} K_{s}(y, Y ; 0) .
$$

found by solving a series of Homologic equations ${ }^{1}$ :

$$
\left[H_{0} ; W_{s}\right]+\tilde{H}_{s}=K_{s} \quad \forall s \geq 1
$$

where the terms $\tilde{H}_{s}$ are found by:

$$
\begin{aligned}
& \tilde{H}_{1}:=H_{1} \\
& \tilde{H}_{s}=\tilde{H}_{s-1,1}+\left[\tilde{H}_{s-1,0}+\left[H_{0} ; W_{s-1}\right] ; W_{1}\right] \quad \forall s \geq 2
\end{aligned}
$$

with

$$
\left\{\begin{array}{l}
\tilde{H}_{1,0}:=\tilde{H}_{1}:=H_{1} \\
\tilde{H}_{i, s-i}=H_{s}+\sum_{j=0}^{s-2}\left(\begin{array}{c}
s-i \\
j
\end{array}\right)\left[H_{s-j-i} ; W_{j+1}\right] \quad \text { if } \quad i=1, s \geq 2 \\
\tilde{H}_{i, s-i}=\tilde{H}_{i-1, s-i+1}+\sum_{j=0}^{s-i}\left(\begin{array}{c}
s-i \\
j
\end{array}\right)\left[\tilde{H}_{i-1, s-j-i}+\left[H_{0} ; W_{s-j-1}\right] ; W_{j+1}\right] \\
\quad \text { if } i \neq 1, s \geq 2
\end{array}\right.
$$

This series of homological equations can be seen as both

- a way to find the coefficients of the transformed Hamiltonian given a generating function of a Lie transformation

- a way to find the generating function of the Lie transformation that maps the initial Hamiltonian into a prescribed one.

The relegation and the normalization algorithms (see Deprit et al. (2001) and Deprit (1982) respectively) are two different methods of solving such homological equations. The normalization is the Lie transformation that maps a Hamiltonian $H=\sum_{s \geq 0} \frac{\epsilon^{s}}{s !} H_{s}$ into an equivalent one which admits the principal term $H_{0}$ as integral of the transformed system Deprit et al. (1969). For the relegation, instead, the criteria for selecting the elements of the transformed Hamiltonian $K=K_{0}+\sum_{s \geq 1} \frac{\epsilon^{s}}{s !} K_{s}=K_{0}+\sum_{s \geq 1} \frac{\epsilon^{s}}{s !}\left(\sum_{j=0}^{p}\left(K_{s, p}\right)+R_{s}\right)$ are based only on a part

${ }^{1}$ Let $\mathrm{A}$ be an open subset of $C^{n} \times C^{n}$. If the mappings $f(X, x)$ and $g(X, x)$ from $A$ to $C$ are differentiable in $A$, the Poisson bracket of $f$ and $g([f ; g])$, in that order, is the mapping from $A$ to $C([f ; g]: A \rightarrow C)$ that maps $(X, x) \rightarrow D_{2} f(X, x) \cdot D_{1} g(X, x)-D_{1} f(X, x) \cdot D_{2} g(X, x)$. 
of the principal term $H_{0}$, which becomes the integral of a part of the transformed Hamiltonian $\tilde{K}=K_{0}+\sum_{s \geq 1} \frac{\epsilon^{s}}{s !} \tilde{K}_{s}=K_{0}+\sum_{s \geq 1} \frac{\epsilon^{s}}{s !}\left(\sum_{j=0}^{p} K_{s, p}\right)$.

\section{The relegation of the argument of node $\nu$}

\subsection{Theory}

For the case of fast rotating asteroids, the algorithm described in Deprit et al. (2001) is here applied find a transformation of coordinates which maps the Hamiltonian into a new one where the action of the variable $\nu$ is "relegated" to a certain degree $p_{\max }$, namely in the transformed Hamiltonian $\nu$ will only appear in terms of degree $\sim O\left(\frac{1}{r^{p} \max }\right)$.

To this end the Hamiltonian (4) is rearranged as a power series in the generic small parameter $\epsilon$

$$
H=H_{0}+\epsilon H_{1}
$$

with:

$$
\begin{aligned}
& H_{0}=\frac{1}{2}\left(R^{2}+\frac{\Theta^{2}}{r^{2}}\right)-\frac{M \mathcal{G}}{r}-\omega N \\
& H_{1}=U(r, \theta, \nu)
\end{aligned}
$$

Where

$$
\begin{aligned}
U(r, \theta, \nu) & =-\frac{\mathcal{G M}}{r \epsilon} \sum_{n=1}^{\infty}\left(\frac{\alpha}{r}\right)^{n} \sum_{m=0}^{n} \sum_{j=-n}^{n}\left(\mathcal{G}_{n, m, j}^{1}(I) \cos (m \nu-j \theta)+\right. \\
& \left.+\mathcal{G}_{n, m, j}^{2}(I) \sin (m \nu-j \theta)\right)
\end{aligned}
$$

and $\mathcal{G}_{n, m, j}^{1}(I)$ and $\mathcal{G}_{n, m, j}^{2}(I)$ as in $(19)$ and $\overline{\mathcal{G}}_{n, m, j}(I)$ as in (16).

This asymptotic expansion of the Hamiltonian is equivalent to stating that Keplerian term plus the Coriolis force dominate the dynamics and to leave the hierarchy of the remaining terms of the inhomogeneous potential, all set to the same order, to be decided by the relative influence of their corresponding spherical harmonic coefficients.

The principal term is then divided as a sum $H_{0}=H_{K}+G$ (Keplerian+Coriolis forces), with:

$$
\begin{aligned}
& H_{K}:=\frac{1}{2}\left(R^{2}+\frac{\Theta^{2}}{r^{2}}\right)-\frac{M \mathcal{G}}{r} \\
& G=-\omega N
\end{aligned}
$$

and where $\left[H_{K} ; G\right]=0$.

Moreover, as we are in the fast rotating case it results $|G|>\left|H_{K}\right|$. Let $W(X, x)$ be a differentiable mapping of $A$ into $C$. The Lie derivative $\mathcal{L}_{W}$ induced by $W$ is the operator from $A$ to $C L_{W}: A \rightarrow C$ that maps any function $f(X, x)$ into its Poisson Bracket with $W$, namely $f(X, x): \rightarrow[f ; W]$. It must be noted that $\mathcal{L}_{G}$ is semi-simple over a Poisson algebra of functions $P$, where $\mathcal{L}_{G}$ is the Lie derivative with respect to $G$.

The relegation algorithm maps the Hamiltonian (23) into an equivalent one of the form:

$$
K=K_{0}+\sum_{s \geq 1} \frac{\epsilon^{s}}{s !} K_{s}=\sum_{s \geq 0} \frac{\epsilon^{s}}{s !}\left(\sum_{j=0}^{p}\left(K_{s, p}\right)+R_{s}\right)
$$


with $K_{0}=H_{0}(y, Y)$ and the coefficients $K_{s, p} \in \operatorname{ker}\left(\mathcal{L}_{G}\right)$.

In contrast with normalization, the term $K_{s}$ may not belong to $\operatorname{ker}\left(\mathcal{L}_{G}\right)$ due to the presence of the residual $R_{s}$. In such Hamiltonian the action of $\nu$ is relegated to the reminder $R_{s} \sim O\left(\frac{1}{r^{p}+2}\right)$ since $[K ; G]=\left[H_{0} ; G\right]+[R ; G]$.

As a result the truncated system

$$
K=\sum_{s \geq 0} \frac{\epsilon^{s}}{s !} \sum_{j=0}^{p} K_{s, p},
$$

is considered, which approximate the starting Hamiltonian and admits $G$ as an integral.

\subsection{Algorithm}

The general relegation algorithm is briefly described here before the application to the problem. For each homologic equation:

$$
\left[H_{0} ; W_{s}\right]+\tilde{H}_{s}=K_{s}
$$

considering that, as $\mathcal{L}_{G}$ is semi-simple, there $\exists K_{s, 0}, W_{s, 0} \in P$ s.t.

$$
\left\{\begin{array}{l}
\tilde{H}_{s}=K_{s, 0}+\left[W_{s, 0} ; G\right] \\
K_{s, 0} \in \operatorname{Ker}\left(\mathcal{L}_{G}\right) .
\end{array}\right.
$$

Therefore (29) becomes:

$$
\left[H_{0} ; W_{s}\right]+\left[W_{s, 0} ; G\right]=K_{s}-K_{s, 0} .
$$

Thus, setting $W_{s}=W_{s, 0}^{*}+W_{s, 0},(31)$ yields:

$$
\left[H_{0} ; W_{s}^{*}\right]+\left[H_{0}-G ; W_{s, 0}\right]=K_{s}-K_{s, 0} .
$$

The algorithm continues re-invoking $p$-times the semi-simplicity of $\mathcal{L}_{G}$, and finding $\forall 1 \leq j \leq p \quad K_{s, j}, W_{s, j} \in P$ s.t.

$$
\left\{\begin{array}{l}
{\left[H_{0}-G ; W_{s, j-1}\right]=K_{s, j}+\left[W_{s, j} ; G\right]} \\
K_{s, j} \in \operatorname{Ker}\left(\mathcal{L}_{G}\right)
\end{array}\right.
$$

and setting $p$-times $\forall 1 \leq j \leq p \quad W_{s, j-1}=W_{s, j}^{*}+W_{s, j}$.

Finally the algorithm ends at a certain iteration $p$ setting $W_{s, p}^{*}=0$ and obtaining (32) to become:

$$
K_{s}=\sum_{j=0}^{p}\left(K_{s, j}\right)+R_{s}
$$

with $R_{s}:=\left[H_{0}-G ; W_{s, p}\right]$ Although the procedure is general, as in the previous literature (see San-Juan et al. (2002), or Scheeres et al. (1998)), for the relegation as well as for the normalization, only the first Homologic equation will here be considered and explicitly evaluated. 


\subsection{Application}

Following (San-Juan et al. (2002)), the Relegation algorithm is now applied to the Hamiltonian (23). By (26), it is first noted that

$$
\left[H_{K} ; \cdot\right]=\left[\frac{1}{2}\left(R^{2}+\frac{\Theta^{2}}{r^{2}}\right)-\frac{M \mathcal{G}}{r} ; \cdot\right]=R \frac{\partial \cdot}{\partial r}+\frac{\Theta}{r^{2}} \frac{\partial \cdot}{\partial \theta}-\left(\frac{\Theta^{2}}{r^{3}}-\frac{M \mathcal{G}}{r^{2}}\right) \frac{\partial \cdot}{\partial R}
$$

and that

$$
[\cdot ; G]=[\cdot ;-\omega N]=-\omega \frac{\partial \cdot}{\partial \nu}
$$

Note that, as the only variable in $G$ is $N$, the Poisson brackets essentially "degenerate" into a derivative in the argument of node $\nu$ which means that the "inverse" of the Poisson brackets, in this case, degenerate into an integral over the fast angle $\nu$. Therefore, in this case, the relegation is equivalent to taking the average over the argument of node.

Now, considering (26) and (21), the first homologic equation (29) becomes:

$$
\left[H_{0} ; W_{1}\right]+U=K_{1}
$$

with $U$ as in (25).

FIRST ITERATION

Thus, following (30), by (35) and (36), the first iteration consists in finding $K_{1,0}$ and $W_{1,0}$ such that

$$
\left\{\begin{array}{l}
U=K_{1,0}-\omega \frac{\partial W_{1,0}}{\partial \nu} \\
\frac{\partial K_{1,0}}{\partial \nu}=0
\end{array}\right.
$$

Therefore $K_{1,0}$ is the collection of all the terms of $U$ such that their derivative with respect to $\nu$ is zero, i.e. that do not depend on $\nu$ :

$$
K_{1,0}=-\frac{\mathcal{G} M}{r \epsilon} \sum_{n=1}^{\infty}\left(\frac{\alpha}{r}\right)^{n} \sum_{j=-n}^{n}\left(\mathcal{G}_{n, 0, j}^{1}(I) \cos (-j \theta)+\mathcal{G}_{n, 0, j}^{2}(I) \sin (-j \theta)\right)
$$

Then, inverting (38):

$$
\begin{aligned}
W_{1,0}=- & \frac{1}{\omega} \int\left(U-K_{1,0}\right) d \nu \\
=- & \frac{1}{\omega} \int\left(-\frac{\mathcal{G} M}{r \epsilon} \sum_{n=1}^{\infty}\left(\frac{\alpha}{r}\right)^{n} \sum_{m=1}^{n} \sum_{j=-n}^{n}\left(\mathcal{G}_{n, m, j}^{1}(I) \cos (m \nu-j \theta)+\right.\right. \\
& \left.\left.\quad+\mathcal{G}_{n, m, j}^{2}(I) \sin (m \nu-j \theta)\right)\right) d \nu \\
= & +\frac{\mathcal{G} M}{r \omega \epsilon} \sum_{n=1}^{\infty}\left(\frac{\alpha}{r}\right)^{n} \sum_{m=1}^{n} \sum_{j=-n}^{n} \frac{1}{m}\left(\mathcal{G}_{n, m, j}^{1}(I) \sin (m \nu-j \theta)+\right. \\
& \left.\quad \mathcal{G}_{n, m, j}^{2}(I) \cos (m \nu-j \theta)\right)
\end{aligned}
$$

which is periodic in $\nu$.

SECOND ITERATION

After the first iteration equation (32) becomes:

$$
\left[H_{0} ; W_{1}^{*}\right]+\left[H_{0}-G ; W_{1,0}\right]=K_{1}-K_{1,0} .
$$


where from (33) and (40):

$$
\begin{aligned}
{\left[H_{0}-\right.} & \left.G ; W_{1,0}\right]=R \frac{\partial W_{1,0}}{\partial r}+\frac{\Theta}{r^{2}} \frac{\partial W_{1,0}}{\partial \theta}-\left(\frac{\Theta^{2}}{r^{3}}-\frac{M \mathcal{G}}{r^{2}}\right) \frac{\partial W_{1,0}}{\partial R} \\
= & -\frac{\mathcal{G} R M}{r^{2} \omega \epsilon} \sum_{n=1}^{\infty}(n+1)\left(\frac{\alpha}{r}\right)^{n} \sum_{m=1}^{n} \sum_{j=-n}^{n} \frac{1}{m}\left(\mathcal{G}_{n, m, j}^{1}(I) \sin (m \nu-j \theta)+\right. \\
& \left.-\mathcal{G}_{n, m, j}^{2}(I) \cos (m \nu-j \theta)\right)+ \\
& -\frac{\mathcal{G} \Theta M}{r^{3} \omega \epsilon} \sum_{n=1}^{\infty}\left(\frac{\alpha}{r}\right)^{n} \sum_{m=1}^{n} \sum_{j=-n}^{n} \frac{j}{m}\left(\mathcal{G}_{n, m, j}^{1}(I) \cos (m \nu-j \theta)+\right. \\
& \left.+\mathcal{G}_{n, m, j}^{2}(I) \sin (m \nu-j \theta)\right)
\end{aligned}
$$

and which is still $\nu$-periodic.

Following 33, the second iteration consists in finding $K_{1,1}$ and $W_{1,1}$ such that:

$$
\left\{\begin{array}{l}
{\left[H_{0}-G ; W_{1,0}\right]=K_{1,1}-\omega \frac{\partial W_{1,1}}{\partial \nu}} \\
\frac{\partial K_{1,1}}{\partial \nu}=0
\end{array}\right.
$$

with $\left[H_{0}-G ; W_{1,0}\right]$ as in (42).

Thus $K_{1,1}$ is the collection of all the terms of $\left[H_{0}-G ; W_{1,0}\right]$ such that their derivative with respect to $\nu$ is zero, i.e. that do not depend on $\nu$, which implies that $K_{1,1}=0$.

Then, inverting (43), yields:

$$
\begin{aligned}
W_{1,1}= & -\frac{1}{\omega} \int\left[H_{0}-G ; W_{1,0}\right] d \nu \\
= & -\frac{\mathcal{G} R M}{r^{2} \omega^{2} \epsilon} \sum_{n=1}^{\infty}(n+1)\left(\frac{\alpha}{r}\right)^{n} \sum_{m=1}^{n} \sum_{j=-n}^{n} \frac{1}{m^{2}}\left(\mathcal{G}_{n, m, j}^{1}(I) \cos (m \nu-j \theta)+\right. \\
& \left.+\mathcal{G}_{n, m, j}^{2}(I) \sin (m \nu-j \theta)\right)+ \\
& +\frac{\mathcal{G} \Theta M}{r^{3} \omega^{2} \epsilon} \sum_{n=1}^{\infty}\left(\frac{\alpha}{r}\right)^{n} \sum_{m=1}^{n} \sum_{j=-n}^{n} \frac{j}{m^{2}}\left(\mathcal{G}_{n, m, j}^{1}(I) \sin (m \nu-j \theta)+\right. \\
& \left.-\mathcal{G}_{n, m, j}^{2}(I) \cos (m \nu-j \theta)\right)
\end{aligned}
$$

\section{OTHER ITERATIONS}

In complete analogy with the second iteration of the relegation it is found that $\forall 2 \leq j \leq p$ :

$$
\begin{aligned}
& K_{1, j}=0 \\
& W_{1, j}=-\frac{1}{\omega} \int\left[H_{0}-G ; W_{1, j-1}\right] d \nu
\end{aligned}
$$

The explicit expression for all the further iterations terms of the generator, which have been used for the applications, have been found symbolically with a script coded in the software Mathematica. 


\subsection{Section Summary}

As in (34) the relegation is iterated $p-1$ times and, setting $W_{1, p}^{*}=0$, yields:

$$
\begin{aligned}
W_{1} & =\sum_{j=0}^{p-1} W_{1, j} \\
K_{1} & =\sum_{j=0}^{p-1} K_{1, j}+R_{1}=K_{1,0}+R_{1}
\end{aligned}
$$

with

$$
R_{1}=\left[H_{0}-G ; W_{1, p}\right]
$$

with $K_{1,0}$ as in (39).

It must be noted that, at each step of relegation $1 \leq j \leq p$ the coefficient $W_{1, j}$ of the generator is $O\left(\frac{1}{r^{j+2}}\right)$ which implies that when the algorithm is stopped at the $p^{\text {th }}$ iteration the "remainder"

$$
\left[H_{0}-G ; W_{1, p}\right]=R \frac{\partial W_{1, p}}{\partial r}+\frac{\Theta}{r^{2}} \frac{\partial W_{1, p}}{\partial \theta}-\left(\frac{\Theta^{2}}{r^{3}}-\frac{M \mathcal{G}}{r^{2}}\right) \frac{\partial W_{1, p}}{\partial R}
$$

is of degree $O\left(\frac{1}{r^{p+3}}\right)$.

When applying this algorithm to a real asteroid, only a finite number of spherical harmonic coefficients will be known, that is $\exists n_{\max }$ such that we know all the $C_{n, m}$ and $S_{n, m}$ up to $C_{n_{\max }, n_{\max }}$ and $S_{n_{\max }, n_{\max }}$ included. This, by (25), implies that in the potential the terms of degree $O\left(\frac{1}{r^{(n \max +2)}}\right)$ and higher have been truncated; thus, in the relegation algorithm the maximum number of iterations $p$ is set to $n_{\max }-1$ such that the reminder of the algorithm $O\left(\frac{1}{r^{p+3}}\right)=$ $O\left(\frac{1}{r^{n} \max +2}\right)$ can be dropped and the Hamiltonian becomes

$$
\begin{aligned}
K & =H_{0}+\epsilon K_{1,0} \\
& =\frac{1}{2}\left(R^{2}+\frac{\Theta^{2}}{r^{2}}\right)-\frac{M \mathcal{G}}{r}-\omega N+\epsilon K_{1,0}
\end{aligned}
$$

with $K_{1,0}$ as in (39).

Moreover the resulting Hamiltonian is equivalent to the one in the main problem of the artificial satellite, in which the argument of node $\nu$ is cyclic, the coriolis term $-\omega N$ is constant and can be neglected from the Hamiltonian. Therefore a closed form Delaunay normalization can be performed, for a further reduction of the degrees of freedom, thus yielding an integrable Hamiltonian.

\section{Delaunay Coordinates}

In order to perform the Delaunay normalisation the Hamiltonian must be transformed from the relegated Wittaker variables to the Delaunay coordinates. The Delaunay coordinates are symplectic action-angle variables $(L, G, H, \ell, g, h)$, where the angles $\ell, g$ and $h$ are conjugate to the actions $L, G$ and $H$ respectively, where

Note that fixing the maximum degree $n_{\max }$ of terms for the potential means that all the terms up to $C_{n \max , n \max }$ and $S_{n \max , n \max }$ are taken into account, which is a total of $(n \max +2)(n \max +1)$ coefficients of the potential 
- $\ell$ is the mean anomaly measured from the pericenter;

- $g$ is the argument of the pericenter;

- $h$ is the longitude of the nodes;

- $L$ is related to the semi-major axis, $a$, by $L=\sqrt{\mathcal{G M a}}$;

- $G$ is the total angular momentum of the spacecraft with respect to the Asteroid (in the inertial frame), related to the eccentricity and the variable $L$ by $e=\sqrt{1-\frac{G^{2}}{L^{2}}}$;

- $H$ is the $z$-component of the total angular momentum, i.e. $H=G \cos I$.

Moreover the relation between the True anomaly $f$ and the Eccentric anomaly $u$ is defined as:

$$
\tan \left(\frac{f}{2}\right)=\sqrt{\frac{1+e}{1-e}} \tan \left(\frac{u}{2}\right),
$$

which, in particular, implies

$$
r=a(1-e \cos u)=a \frac{1-e^{2}}{1+e \cos f} .
$$

A quick derivation of such coordinates is here provided, while a full derivation can be found in Arnol'd (2006) and Celletti and Chierchia (2007) (also see Goldstein et al (2001), Stiefel and Scheifele (1971)).

The change of coordinates which takes from the Wittaker variables to the Delaunay coordinates is generated by the function

$$
S=\int_{r_{-}\left(-\frac{(\mathcal{G} M)^{2}}{L^{2}}\right)}^{r} \sqrt{-\frac{G^{2}}{r^{2}}+2 \frac{M \mathcal{G}}{r}-\frac{(\mathcal{G} M)^{2}}{L^{2}}} d \rho+G(\theta-f)+H \nu
$$

It is completely canonical as

$$
d \ell \wedge d L+d g \wedge d G+d h \wedge d H=d r \wedge d R+d \theta \wedge d \Theta+d \nu \wedge d N
$$

that is:

$$
\begin{aligned}
& R=\frac{\partial S}{\partial r}=\sqrt{-\frac{\Theta^{2}}{r^{2}}+2 \frac{M \mathcal{G}}{r}-\frac{(\mathcal{G} M)^{2}}{L^{2}}} \\
& \Theta=\frac{\partial S}{\partial \theta}=G \\
& N=\frac{\partial S}{\partial \nu}=H \\
& \ell=\frac{\partial S}{\partial L}=\ldots=u-e \sin u \\
& g=\frac{\partial S}{\partial G}=(\theta-f) \\
& h=\frac{\partial S}{\partial H}=\nu .
\end{aligned}
$$

Plus, by previous results we know that

$$
N=G \cos I \quad \Rightarrow \quad H=G \cos I .
$$

The relegated Hamiltonian (49) in the Delaunay coordinates, by (51), takes the form

$$
J=J_{0}+\epsilon J_{1}
$$


with:

$$
\begin{aligned}
J_{0}= & -\frac{(\mathcal{G} M)^{2}}{2 L^{2}} \\
J_{1}= & -\frac{\mathcal{G} M}{\epsilon} \sum_{n=1}^{\infty} \alpha^{n}\left(\frac{(1+e \cos f)}{\left(a\left(1-e^{2}\right)\right)}\right)^{n+1} \sum_{j=-n}^{n}\left(\mathcal{G}_{n, 0, j}^{1}(G, H) \cos (-j(f+g))+\right. \\
& \left.+\mathcal{G}_{n, 0, j}^{2}(G, H) \sin (-j(f+g))\right)
\end{aligned}
$$

\subsection{The Normalization algorithm}

The closed form normalization algorithm (Deprit (1982)) is briefly illustrated here, which, instead of using the expansions of $r$ and $f$ in powers of the eccentricity, changes the independent variable from time to the true anomaly $f$.

\section{Definition 3}

A formal series $K^{\prime}(y, Y, \epsilon)=\sum_{s=0}^{\infty} \frac{\epsilon^{s}}{s !} K_{s}^{\prime}(y, Y)$ is said to be in Delaunay normal form if the Lie derivative $L_{K_{0}^{\prime}} J$ is zero, that is $\left[K_{s}^{\prime}, K_{0}^{\prime}\right]=0 \quad \forall s \geq 0$.

In our case, as $K_{0}^{\prime}=J_{0}=-\frac{(\mathcal{G} M)^{2}}{2 L^{2}}$, the Lie derivative

$$
L_{K_{0}^{\prime}}(\cdot)=\frac{(\mathcal{G} M)^{2}}{L^{3}} \frac{\partial(\cdot)}{\partial \ell}
$$

therefore the new Hamiltonian (56) will be in normal form if and only if

$$
\frac{\partial K_{1}^{\prime}}{\partial \ell}=0
$$

Note that, as in (36) for the relegation, the normalization degenerates into an average over the mean anomaly $\ell$.

\subsection{Algorithm}

As in (29), remembering that $\tilde{K}_{1}^{\prime}=J_{1}$, we set the first homologic equation:

$$
\begin{aligned}
& {\left[J_{0} ; \bar{W}_{1}\right]+\tilde{K}_{1}^{\prime}=K_{1}^{\prime}} \\
& \Leftrightarrow-\frac{(\mathcal{G} M)^{2}}{L^{3}} \frac{\partial W_{1}^{\prime}}{\partial \ell}+J_{1}=K_{1}^{\prime}
\end{aligned}
$$

Now, as we want $K_{1}^{\prime}$ to be in Delaunay normal form $\left(\Leftrightarrow \frac{\partial K_{1}^{\prime}}{\partial \ell}=0\right)$, we set

$$
K_{1}^{\prime}=\frac{1}{2 \pi} \int_{0}^{2 \pi} J_{1} d \ell
$$

This integral is solved by changing the independent variable from $\ell$ to be the true anomaly $f$ by the relation

$$
\frac{d f}{d \ell}=\frac{d f}{d u} \frac{d u}{d \ell}=\left(\frac{1+e \cos f}{\sqrt{1-e^{2}}}\right)\left(\frac{1}{1-e \cos u}\right)=\frac{a^{2} \sqrt{1-e^{2}}}{r^{2}}
$$


Finally, inverting (58), yields the first order generating function:

$$
W_{1}^{\prime}=\int \frac{L^{3}}{(\mathcal{G} M)^{2}}\left(J_{1}-\frac{1}{2 \pi} \int_{0}^{2 \pi} J_{1} d \ell\right) d \ell
$$

This leads to an integrable, two degree of freedom, Hamiltonian which approximates the first homologic equation of the system which includes arbitrary degree spherical harmonic coefficients. This approximated system can now be applied to every inhomogeneous body in order to determine possible orbits useful for scientific observation missions such as frozen orbits.

Again, in this paper, only the first Homologic equation is considered and explicitly evaluated and the explicit expressions of (59) and (61) used for the applications have been obtained using a script in the Mathematica software.

Notice that, restricting the result to the case where all the $S_{n, m}$ and $C_{n, m}$ coefficients are zero except for the ellipticity and oblateness terms, the resulting Hamiltonian is reduced to that obtained by San-Juan et al. (2002) and Scheeres et al. (1998).

However, with respect to the Hamiltonian obtained in these papers, it must be highlighted that, considering arbitrary degree of spherical harmonic coefficients, the resulting Hamiltonian will, in general, contain both the relegated variables $G$ and $g$, thus the system is still integrable but the solution cannot be explicitly solved, i.e. it is no longer "trivially integrable" as in San-Juan et al. (2002).

\section{Applications and conclusions}

The Hamiltonian obtained is of the form: $K^{\prime}\left(L, G, H,{ }_{-}, g,_{-}\right)$thus the equations of motion are:

$$
\begin{aligned}
& \ell^{\prime}(t)=\frac{\partial K^{\prime}}{\partial L} \\
& g^{\prime}(t)=\frac{\partial K^{\prime}}{\partial G} \\
& h^{\prime}(t)=\frac{\partial K^{\prime}}{\partial H} \\
& L^{\prime}(t)=0 \\
& G^{\prime}(t)=-\frac{\partial K^{\prime}}{\partial g} \\
& H^{\prime}(t)=0,
\end{aligned}
$$

where $L$ and $H$ are constants and all the other motions will only depend on $G(t)$ and $g(t)$.

It is evident that in a frozen orbit $L, G, H, g, a, e, I$ are constant.

For the properties of the Lie transformations, the "normalized" eccentricity, inclination and argument of perigee are related to their relative "real" equivalents by the generator of the transformation (see Deprit (1969)), and can thus be interpreted as a perturbed version of their real correspondents.

Results show that these initial conditions can be used in the initial system describing the full dynamics (the one described by the Hamiltonian (4)) to generate a good initial guess for frozen orbits around any inhomogeneous body.

Three examples of the resulting frozen orbits are shown for the asteroid 433-Eros. This highly irregular, elongated, Near Earth Asteroid, is the main example used in the literature for the inhomogeneous gravity field. For this the spherical harmonic coefficients can be found up to the $15^{\text {th }}$ degree, i.e. 272 


\begin{tabular}{|c|c|}
\hline $\begin{array}{c}\text { Mass } \\
\mathrm{kg}\end{array}$ & $6.6904 \times 10^{15}$ \\
\hline $\begin{array}{c}\text { Rotational velocity } \\
\mathrm{rad} / \mathrm{s}\end{array}$ & $3.31182 \times 10^{-4}$ \\
\hline $\begin{array}{c}\text { Reference Radius } \\
\mathrm{km}\end{array}$ & 16 \\
\hline
\end{tabular}

Table 1: Physical properties of 433-Eros

coefficients (see Appendix A). Some physical properties of 433-Eros are shown in Table 1.

For illustration purposes three different triples of initial parameters, eccentricity $E_{0}$, inclination $I_{0}$ and argument of perigee $g_{0}$ are fixed. Each triple yields the initial conditions $f_{0}, h_{0}, L_{0}, G_{0}$ and $H_{0}$ for the corresponding frozen orbit found. The results obtained for 433-Eros are collected in Table 2. In the last row of the table, the initial semimajor axis $a_{0}$ of the resulting orbits has also been recorded.

\begin{tabular}{|c|c|c|c|}
\hline & Fig. 1 & Fig. 2 & Fig. 3 \\
& $I_{0}=0.5$ & $I_{0}=1.1$ & $I_{0}=0.001$ \\
& $E_{0}=0.001$ & $E_{0}=0.4$ & $E_{0}=0.5$ \\
& $g_{0}=-\pi / 2$ & $g_{0}=-\pi / 2$ & $g_{0}=\pi / 2$ \\
\hline$h_{0}$ & $\pi$ & $\pi$ & $\pi$ \\
\hline$f_{0}$ & $\pi$ & $\pi$ & $\pi$ \\
\hline \hline$G_{0}$ & 234.612 & 187.656 & 302.438 \\
\hline$L_{0}$ & 234.612 & 204.749 & 504.063 \\
\hline$H_{0}$ & 205.892 & 85.119 & 302.437 \\
\hline \hline$a_{0}$ & $\sim 100$ & $\sim 90 \mathrm{~km}$ & $\sim 500 \mathrm{~km}$ \\
\hline
\end{tabular}

Table 2: 433-Eros: initial conditions for frozen orbits 
The resulting orbits for 433-Eros, in the cartesian inertial frame of reference centered in the center of mass of the inhomogeneous body:

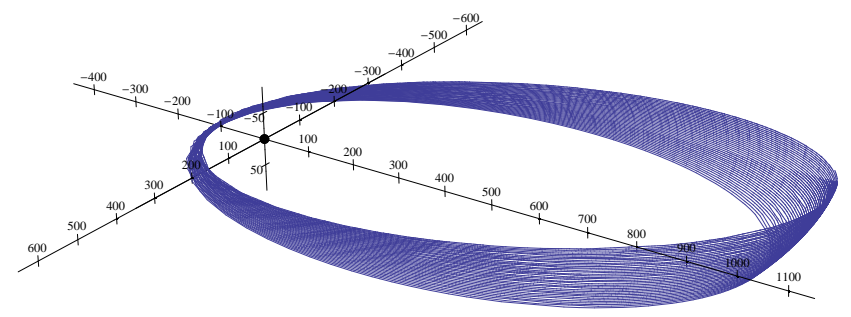

Figure 3: 433-Eros: $I_{0}=0.5, E_{0}=0.001, g_{0}=-\pi / 2$ : after 5 years

\section{Acknowledgements}

Part of this work has been developed in collaboration with the Advanced Concepts Team (ACT) at ESTEC funded from the European Space Agency (ESA) as an Ariadna Study Project. 


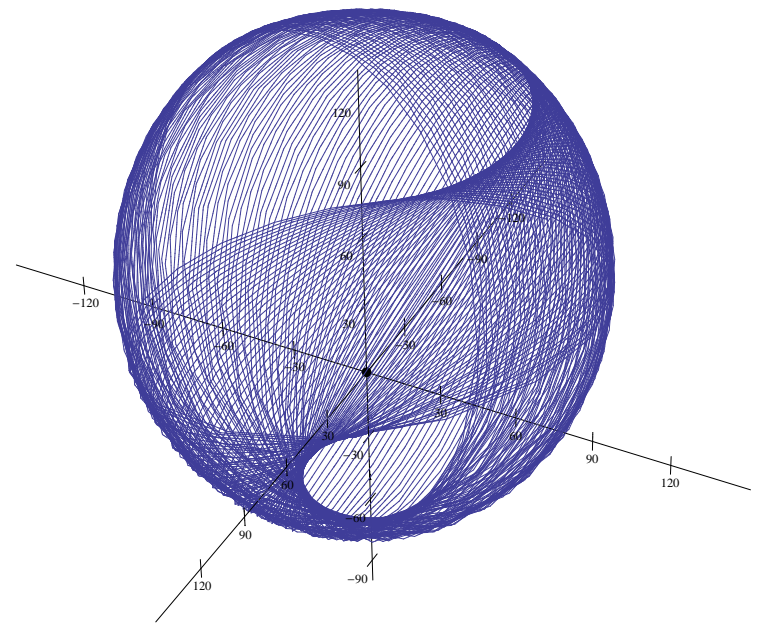

Figure 4: 433-Eros: $I_{0}=1.1, E_{0}=0.4, g_{0}=-\pi / 2$ : after 5 years 


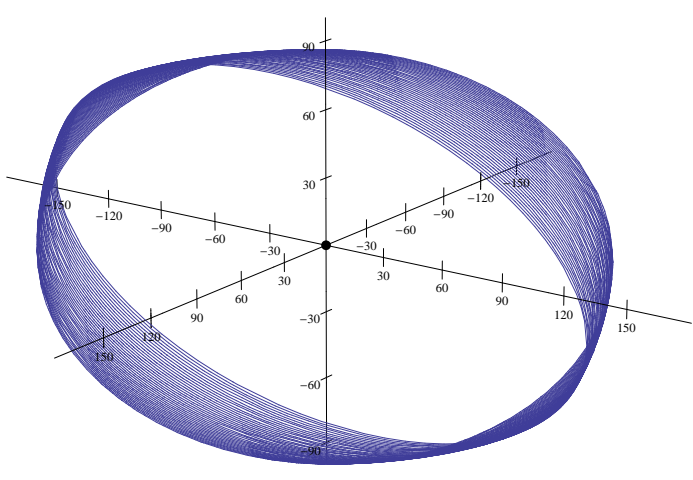

Figure 5: 433-Eros: $I_{0}=0.001, E_{0}=0.5, g_{0}=\pi / 2$ : after 5 years 


\section{Appendix A}

The un-normalized spherical harmonic coefficients of 433-Eros are here listed. This coefficients are the harmonic coefficients gravity solution NEAR15A, a 15 th degree and order model obtained from radiometric tracking (Doppler and range data) and landmark tracking of the NEAR spacecraft in orbit about Eros. The gravity model includes data from the entire mission beginning with orbit insertion on Feb. 14, 2000 and ending with the first descent maneuver for landing on Feb. 12, 2001

\begin{tabular}{|c|c|}
\hline$C_{0,0}$ & 1 \\
\hline$C_{1,0}$ & 0 \\
\hline$C_{1,1}$ & 0 \\
\hline$C_{2,0}$ & $-1.65899 \times 10^{-1}$ \\
\hline$C_{2,1}$ & $-2.11454 \times 10^{-6}$ \\
\hline$C_{2,2}$ & $5.31886 \times 10^{-2}$ \\
\hline$C_{3,0}$ & $-5.29244 \times 10^{-3}$ \\
\hline$C_{3,1}$ & $4.38548 \times 10^{-3}$ \\
\hline$C_{3,2}$ & $6.0659 \times 10^{-4}$ \\
\hline$C_{3,3}$ & $-1.4525 \times 10^{-3}$ \\
\hline$C_{4,0}$ & $5.48636 \times 10^{-2}$ \\
\hline$C_{4,1}$ & $-9.52013 \times 10^{-5}$ \\
\hline$C_{4,2}$ & $-3.90614 \times 10^{-3}$ \\
\hline$C_{4,3}$ & $-1.79405 \times 10^{-5}$ \\
\hline$C_{4,4}$ & $3.68808 \times 10^{-4}$ \\
\hline$C_{5,0}$ & $3.09067 \times 10^{-3}$ \\
\hline$C_{5,1}$ & $-2.36787 \times 10^{-3}$ \\
\hline$C_{5,2}$ & $-1.26781 \times 10^{-4}$ \\
\hline$C_{5,3}$ & $1.51169 \times 10^{-4}$ \\
\hline$C_{5,4}$ & $3.86908 \times 10^{-6}$ \\
\hline$C_{5,5}$ & $-2.51307 \times 10^{-5}$ \\
\hline$C_{6,0}$ & $-2.53848 \times 10^{-2}$ \\
\hline$C_{6,1}$ & $-1.91651 \times 10^{-5}$ \\
\hline$C_{6,2}$ & $8.13891 \times 10^{-4}$ \\
\hline$C_{6,3}$ & $5.9664 \times 10^{-6}$ \\
\hline$C_{6,4}$ & $-2.13764 \times 10^{-5}$ \\
\hline$C_{6,5}$ & $-3.93777 \times 10^{-7}$ \\
\hline$C_{6,6}$ & $1.18484 \times 10^{-6}$ \\
\hline & \\
\hline
\end{tabular}

\begin{tabular}{|c|c|}
\hline$C_{7,0}$ & $-2.50016 \times 10^{-3}$ \\
\hline$C_{7,1}$ & $1.26047 \times 10^{-3}$ \\
\hline$C_{7,2}$ & $3.82038 \times 10^{-5}$ \\
\hline$C_{7,3}$ & $-3.48143 \times 10^{-5}$ \\
\hline$C_{7,4}$ & $-5.15671 \times 10^{-7}$ \\
\hline$C_{7,5}$ & $1.33563 \times 10^{-6}$ \\
\hline$C_{7,6}$ & $2.25518 \times 10^{-9}$ \\
\hline$C_{7,7}$ & $-1.25528 \times 10^{-7}$ \\
\hline$C_{8,0}$ & $1.53478 \times 10^{-2}$ \\
\hline$C_{8,1}$ & $-3.43765 \times 10^{-5}$ \\
\hline$C_{8,2}$ & $-2.57667 \times 10^{-4}$ \\
\hline$C_{8,3}$ & $-3.12096 \times 10^{-6}$ \\
\hline$C_{8,4}$ & $3.61153 \times 10^{-6}$ \\
\hline$C_{8,5}$ & $8.73471 \times 10^{-8}$ \\
\hline$C_{8,6}$ & $-7.09764 \times 10^{-8}$ \\
\hline$C_{8,7}$ & $-9.71194 \times 10^{-10}$ \\
\hline$C_{8,8}$ & $2.89016 \times 10^{-9}$ \\
\hline$C_{9,0}$ & $1.12427 \times 10^{-3}$ \\
\hline$C_{9,1}$ & $-4.97634 \times 10^{-4}$ \\
\hline$C_{9,2}$ & $-2.57824 \times 10^{-5}$ \\
\hline$C_{9,3}$ & $1.07011 \times 10^{-5}$ \\
\hline$C_{9,4}$ & $-4.14388 \times 10^{-7}$ \\
\hline$C_{9,5}$ & $-1.7556 \times 10^{-7}$ \\
\hline$C_{9,6}$ & $-3.11553 \times 10^{-9}$ \\
\hline$C_{9,7}$ & $5.83725 \times 10^{-9}$ \\
\hline$C_{9,8}$ & $1.43792 \times 10^{-10}$ \\
\hline$C_{9,9}$ & $-2.52185 \times 10^{-10}$ \\
\hline$C_{10,0}$ & $-2.23924 \times 10^{-3}$ \\
\hline & \\
\hline
\end{tabular}

\begin{tabular}{|c|c|}
\hline$C_{10,1}$ & $-3.65977 \times 10^{-4}$ \\
\hline$C_{10,2}$ & $8.59725 \times 10^{-5}$ \\
\hline$C_{10,3}$ & $2.44668 \times 10^{-6}$ \\
\hline$C_{10,4}$ & $-2.12904 \times 10^{-8}$ \\
\hline$C_{10,5}$ & $-3.91544 \times 10^{-8}$ \\
\hline$C_{10,6}$ & $1.06018 \times 10^{-8}$ \\
\hline$C_{10,7}$ & $6.6781 \times 10^{-10}$ \\
\hline$C_{10,8}$ & $-1.03388 \times 10^{-10}$ \\
\hline$C_{10,9}$ & $-2.93031 \times 10^{-11}$ \\
\hline$C_{10,10}$ & $4.93363 \times 10^{-12}$ \\
\hline$C_{11,0}$ & $1.04666 \times 10^{-2}$ \\
\hline$C_{11,1}$ & $3.72982 \times 10^{-4}$ \\
\hline$C_{11,2}$ & $3.37686 \times 10^{-6}$ \\
\hline$C_{11,3}$ & $1.80367 \times 10^{-6}$ \\
\hline$C_{11,4}$ & $-5.5386 \times 10^{-7}$ \\
\hline$C_{11,5}$ & $7.57115 \times 10^{-8}$ \\
\hline$C_{11,6}$ & $2.19576 \times 10^{-9}$ \\
\hline$C_{11,7}$ & $5.19815 \times 10^{-11}$ \\
\hline$C_{11,8}$ & $3.75133 \times 10^{-11}$ \\
\hline$C_{11,9}$ & $1.74028 \times 10^{-11}$ \\
\hline$C_{11,10}$ & $-2.76742 \times 10^{-13}$ \\
\hline$C_{11,11}$ & $-2.57971 \times 10^{-13}$ \\
\hline$C_{12,0}$ & $1.71922 \times 10^{-3}$ \\
\hline$C_{12,1}$ & $3.7954 \times 10^{-4}$ \\
\hline$C_{12,2}$ & $1.55553 \times 10^{-4}$ \\
\hline$C_{12,3}$ & $6.86842 \times 10^{-6}$ \\
\hline$C_{12,4}$ & $2.99064 \times 10^{-7}$ \\
\hline$C_{12,5}$ & $-8.38626 \times 10^{-8}$ \\
\hline & \\
\hline
\end{tabular}

\section{References}

Abad, A., Elipe A., Tresaco E.:'Analytical Model to Find Frozen Orbits for a Lunar Orbiter', Journal of Guidance, Control and Dynamics, 32, 888-898, (2009)

Abramowitz, M. and Stegun.,I. A.:' Handbook of Mathematical Functions with Formulas, Graps, and Mathematical Tables', National Bureau Of Standards, Washington (1965) 


\begin{tabular}{|c|c|}
\hline$C_{12,6}$ & $4.07023 \times 10^{-9}$ \\
\hline$C_{12,7}$ & $-1.60746 \times 10^{-10}$ \\
\hline$C_{12,8}$ & $1.86662 \times 10^{-11}$ \\
\hline$C_{12,9}$ & $-4.62122 \times 10^{-12}$ \\
\hline$C_{12,10}$ & $-5.72445 \times 10^{-13}$ \\
\hline$C_{12,11}$ & $2.02689 \times 10^{-14}$ \\
\hline$C_{12,12}$ & $8.12551 \times 10^{-15}$ \\
\hline$C_{13,0}$ & $2.75545 \times 10^{-2}$ \\
\hline$C_{13,1}$ & $-2.9199 \times 10^{-3}$ \\
\hline$C_{13,2}$ & $-2.02593 \times 10^{-6}$ \\
\hline$C_{13,3}$ & $6.84023 \times 10^{-6}$ \\
\hline$C_{13,4}$ & $3.23691 \times 10^{-7}$ \\
\hline$C_{13,5}$ & $-3.54904 \times 10^{-8}$ \\
\hline$C_{13,6}$ & $2.59498 \times 10^{-10}$ \\
\hline$C_{13,7}$ & $4.03437 \times 10^{-11}$ \\
\hline$C_{13,8}$ & $-1.37277 \times 10^{-11}$ \\
\hline$C_{13,9}$ & $-7.31327 \times 10^{-13}$ \\
\hline$C_{13,10}$ & $-7.27471 \times 10^{-14}$ \\
\hline$C_{13,11}$ & $2.30772 \times 10^{-14}$ \\
\hline$C_{13,12}$ & $2.58196 \times 10^{-16}$ \\
\hline$C_{13,13}$ & $-2.18667 \times 10^{-16}$ \\
\hline$C_{14,0}$ & $-1.53377 \times 10^{-2}$ \\
\hline$C_{14,1}$ & $7.66068 \times 10^{-4}$ \\
\hline$C_{14,2}$ & $2.96292 \times 10^{-4}$ \\
\hline$C_{14,3}$ & $5.32869 \times 10^{-6}$ \\
\hline$C_{14,4}$ & $-5.87731 \times 10^{-7}$ \\
\hline$C_{14,5}$ & $-5.31799 \times 10^{-8}$ \\
\hline$C_{14,6}$ & $2.30096 \times 10^{-9}$ \\
\hline & \\
\hline
\end{tabular}

\begin{tabular}{|c|c|}
\hline$C_{14,7}$ & $-7.86604 \times 10^{-11}$ \\
\hline$C_{14,8}$ & $-7.59718 \times 10^{-12}$ \\
\hline$C_{14,9}$ & $-1.0193 \times 10^{-13}$ \\
\hline$C_{14,10}$ & $5.29761 \times 10^{-14}$ \\
\hline$C_{14,11}$ & $7.4175 \times 10^{-15}$ \\
\hline$C_{14,12}$ & $-9.24318 \times 10^{-16}$ \\
\hline$C_{14,13}$ & $-2.2948 \times 10^{-17}$ \\
\hline$C_{14,14}$ & $1.81628 \times 10^{-17}$ \\
\hline$C_{15,0}$ & $2.06404 \times 10^{-2}$ \\
\hline$C_{15,1}$ & $-2.65164 \times 10^{-3}$ \\
\hline$C_{15,2}$ & $9.46812 \times 10^{-6}$ \\
\hline$C_{15,3}$ & $-3.69445 \times 10^{-6}$ \\
\hline$C_{15,4}$ & $3.18757 \times 10^{-7}$ \\
\hline$C_{15,5}$ & $-2.84101 \times 10^{-8}$ \\
\hline$C_{15,6}$ & $-1.9038 \times 10^{-10}$ \\
\hline$C_{15,7}$ & $-6.24463 \times 10^{-11}$ \\
\hline$C_{15,8}$ & $-1.06965 \times 10^{-11}$ \\
\hline$C_{15,9}$ & $-2.61478 \times 10^{-13}$ \\
\hline$C_{15,10}$ & $5.55852 \times 10^{-16}$ \\
\hline$C_{15,11}$ & $-1.64954 \times 10^{-15}$ \\
\hline$C_{15,12}$ & $4.81127 \times 10^{-17}$ \\
\hline$C_{15,13}$ & $2.5553 \times 10^{-17}$ \\
\hline$C_{15,14}$ & $5.60796 \times 10^{-19}$ \\
\hline$C_{15,15}$ & $-5.49434 \times 10^{-19}$ \\
\hline
\end{tabular}

Arnol'd, Vladimir I.; Kozlov, Valery V.; Neishtadt, Anatoly.:' I. Mathematical aspects of classical and celestial mechanics. Dynamical systems. III.' Encyclopaedia of Mathematical Sciences,Springer-Verlag, Berlin ISBN: 978-3-54028246-4; 3-540-28246-7(2006)

Brouwer, D.:" Solution of the Problem of the Artificial Satellite Without Drag' Astronomical Journal, 64 (1959)

Carnelli, Gálvez:'ESA's Don Quijote Mission: an Opportunity for the Investigation of an Artificial Impact Crater on an Asteroid, in Proceedings of the 25th International Symposium on Space Technology and Science, Kanazawa, Japan, (2006) Paper ISTS 2006-k-26.

Celletti, A.; Chierchia, L.:' KAM stability and celestial mechanics.' Mem. Amer. Math. Soc. 187 , no. $878(2007)$

Colombo, C.; Lücking, C., McInnes, C.:" Orbital dynamics of high area-to mass ratio spacecraft with J2 and solar radiation pressure for novel Earth obser- 


\begin{tabular}{|c|c|}
\hline$S_{0,0}$ & 0 \\
\hline$S_{1,0}$ & 0 \\
\hline$S_{1,1}$ & 0 \\
\hline$S_{2,0}$ & 0 \\
\hline$S_{2,1}$ & $-1.80744 \times 10^{-7}$ \\
\hline$S_{2,2}$ & $-1.81446 \times 10^{-2}$ \\
\hline$S_{3,0}$ & 0 \\
\hline$S_{3,1}$ & $3.63836 \times 10^{-3}$ \\
\hline$S_{3,2}$ & $-2.40395 \times 10^{-4}$ \\
\hline$S_{3,3}$ & $-1.68328 \times 10^{-3}$ \\
\hline$S_{4,0}$ & 0 \\
\hline$S_{4,1}$ & $1.29913 \times 10^{-4}$ \\
\hline$S_{4,2}$ & $1.0351 \times 10^{-3}$ \\
\hline$S_{4,3}$ & $-7.12399 \times 10^{-6}$ \\
\hline$S_{4,4}$ & $-1.92384 \times 10^{-4}$ \\
\hline$S_{5,0}$ & 0 \\
\hline$S_{5,1}$ & $-1.04273 \times 10^{-3}$ \\
\hline$S_{5,2}$ & $6.17062 \times 10^{-5}$ \\
\hline$S_{5,3}$ & $1.16925 \times 10^{-4}$ \\
\hline$S_{5,4}$ & $-5.43531 \times 10^{-6}$ \\
\hline$S_{5,5}$ & $-1.43782 \times 10^{-5}$ \\
\hline$S_{6,0}$ & 0 \\
\hline$S_{6,1}$ & $-9.74106 \times 10^{-5}$ \\
\hline$S_{6,2}$ & $-1.48126 \times 10^{-4}$ \\
\hline$S_{6,3}$ & $1.56395 \times 10^{-6}$ \\
\hline$S_{6,4}$ & $1.56395 \times 10^{-6}$ \\
\hline$S_{6,5}$ & $3.86799 \times 10^{-8}$ \\
\hline$S_{6,6}$ & $-3.73278 \times 10^{-7}$ \\
\hline & \\
\hline
\end{tabular}

\begin{tabular}{|c|c|}
\hline$S_{7,0}$ & 0 \\
\hline$S_{7,1}$ & $5.15445 \times 10^{-4}$ \\
\hline$S_{7,2}$ & $-1.97429 \times 10^{-5}$ \\
\hline$S_{7,3}$ & $-2.02322 \times 10^{-5}$ \\
\hline$S_{7,4}$ & $6.94006 \times 10^{-7}$ \\
\hline$S_{7,5}$ & $6.72634 \times 10^{-7}$ \\
\hline$S_{7,6}$ & $-3.44172 \times 10^{-8}$ \\
\hline$S_{7,7}$ & $-4.07766 \times 10^{-8}$ \\
\hline$S_{8,0}$ & 0 \\
\hline$S_{8,1}$ & $-1.24043 \times 10^{-5}$ \\
\hline$S_{8,2}$ & $2.30047 \times 10^{-6}$ \\
\hline$S_{8,3}$ & $-3.22691 \times 10^{-7}$ \\
\hline$S_{8,4}$ & $-6.27617 \times 10^{-7}$ \\
\hline$S_{8,5}$ & $-1.85513 \times 10^{-8}$ \\
\hline$S_{8,6}$ & $6.66802 \times 10^{-10}$ \\
\hline$S_{8,7}$ & $-3.57144 \times 10^{-10}$ \\
\hline$S_{8,8}$ & $1.74786 \times 10^{-9}$ \\
\hline$S_{9,0}$ & 0 \\
\hline$S_{9,1}$ & $-8.17618 \times 10^{-5}$ \\
\hline$S_{9,2}$ & $-1.31237 \times 10^{-5}$ \\
\hline$S_{9,3}$ & $7.54724 \times 10^{-6}$ \\
\hline$S_{9,4}$ & $-2.35188 \times 10^{-7}$ \\
\hline$S_{9,5}$ & $-1.00222 \times 10^{-7}$ \\
\hline$S_{9,6}$ & $1.12056 \times 10^{-9}$ \\
\hline$S_{9,7}$ & $1.6534 \times 10^{-9}$ \\
\hline$S_{9,8}$ & $-2.32921 \times 10^{-11}$ \\
\hline$S_{9,9}$ & $-5.56697 \times 10^{-11}$ \\
\hline$S_{10,0}$ & 0 \\
\hline & \\
\hline
\end{tabular}

\begin{tabular}{|c|c|}
\hline$S_{10,1}$ & $6.94286 \times 10^{-4}$ \\
\hline$S_{10,2}$ & $-4.56443 \times 10^{-5}$ \\
\hline$S_{10,3}$ & $2.62557 \times 10^{-6}$ \\
\hline$S_{10,4}$ & $-4.14985 \times 10^{-7}$ \\
\hline$S_{10,5}$ & $-5.74199 \times 10^{-8}$ \\
\hline$S_{10,6}$ & $6.45742 \times 10^{-9}$ \\
\hline$S_{10,7}$ & $-7.47668 \times 10^{-10}$ \\
\hline$S_{10,8}$ & $-4.99191 \times 10^{-12}$ \\
\hline$S_{10,9}$ & $9.74982 \times 10^{-13}$ \\
\hline$S_{10,10}$ & $5.59573 \times 10^{-12}$ \\
\hline$S_{11,0}$ & 0 \\
\hline$S_{11,1}$ & $-8.17892 \times 10^{-4}$ \\
\hline$S_{11,2}$ & $-6.92074 \times 10^{-5}$ \\
\hline$S_{11,3}$ & $-1.13881 \times 10^{-6}$ \\
\hline$S_{11,4}$ & $-4.84678 \times 10^{-7}$ \\
\hline$S_{11,5}$ & $8.37324 \times 10^{-8}$ \\
\hline$S_{11,6}$ & $-1.09462 \times 10^{-9}$ \\
\hline$S_{11,7}$ & $-2.46115 \times 10^{-10}$ \\
\hline$S_{11,8}$ & $-2.79264 \times 10^{-11}$ \\
\hline$S_{11,9}$ & $9.02775 \times 10^{-12}$ \\
\hline$S_{11,10}$ & $1.31812 \times 10^{-13}$ \\
\hline$S_{11,11}$ & $-1.94565 \times 10^{-13}$ \\
\hline$S_{12,0}$ & 0 \\
\hline$S_{12,1}$ & $1.50676 \times 10^{-3}$ \\
\hline$S_{12,2}$ & $9.64141 \times 10^{-5}$ \\
\hline$S_{12,3}$ & $2.73675 \times 10^{-6}$ \\
\hline$S_{12,4}$ & $-2.39721 \times 10^{-7}$ \\
\hline$S_{12,5}$ & $-3.08972 \times 10^{-8}$ \\
\hline & \\
\hline
\end{tabular}

vation and communication services' Acta Astronautica Vol. 81, Nom. 1, pp. $137150(2012)$

Deprit, A.:'Canonical transformations depending on a small parameter', Celest. Mech. Dyn. Astr., Vol. 1, pp. 12-30 (1969)

Deprit, A., Henrard, J., Price, J. F., Rom, A.:'Birkhoff's Normalization', Boeing Scientific Research Labs Seattle Wash Mathematics Research Lab. (1969)

Deprit, A.:'The main problem of artificial satellite theory for small and moderate eccentricities', Celestial Mechanics and Dynamical Astronomy, 2, 166-206, (1970)

Deprit, A.:'Delaunay Normalizations', Celestial Mechanics and Dynamical Astronomy, 26, 9-21, (1982)

Deprit, A., Palacián, J., Deprit, E.:'The Relegation Algorithm', Celest. Mech. Dyn. Astr., Vol. 79, pp. 157-182 (2001)

Fahnestock, E., Scheeres, D.,:'Dynamical Characterization and Stabilization of Large Gravity-Tractor Designs', Journal of Guidance, Control, and Dynamics, $31,501-521,(2008)$ 


\begin{tabular}{|c|c|}
\hline$S_{12,6}$ & $9.18684 \times 10^{-9}$ \\
\hline$S_{12,7}$ & $-5.56246 \times 10^{-10}$ \\
\hline$S_{12,8}$ & $5.98262 \times 10^{-12}$ \\
\hline$S_{12,9}$ & $1.23035 \times 10^{-13}$ \\
\hline$S_{12,10}$ & $-7.24925 \times 10^{-13}$ \\
\hline$S_{12,11}$ & $1.701 \times 10^{-14}$ \\
\hline$S_{12,12}$ & $1.63895 \times 10^{-14}$ \\
\hline$S_{13,0}$ & 0 \\
\hline$S_{13,1}$ & $-1.24564 \times 10^{-3}$ \\
\hline$S_{13,2}$ & $1.54632 \times 10^{-5}$ \\
\hline$S_{13,3}$ & $-6.74004 \times 10^{-7}$ \\
\hline$S_{13,4}$ & $-1.19607 \times 10^{-6}$ \\
\hline$S_{13,5}$ & $6.26074 \times 10^{-9}$ \\
\hline$S_{13,6}$ & $-1.26688 \times 10^{-10}$ \\
\hline$S_{13,7}$ & $-7.5178 \times 10^{-13}$ \\
\hline$S_{13,8}$ & $-1.60844 \times 10^{-11}$ \\
\hline$S_{13,9}$ & $-9.10394 \times 10^{-14}$ \\
\hline$S_{13,10}$ & $-7.19669 \times 10^{-15}$ \\
\hline$S_{13,11}$ & $-5.20369 \times 10^{-15}$ \\
\hline$S_{13,12}$ & $-1.01803 \times 10^{-16}$ \\
\hline$S_{13,13}$ & $-4.21829 \times 10^{-16}$ \\
\hline$S_{14,0}$ & 0 \\
\hline$S_{14,1}$ & $8.65044 \times 10^{-4}$ \\
\hline$S_{14,2}$ & $1.51562 \times 10^{-4}$ \\
\hline$S_{14,3}$ & $4.31479 \times 10^{-7}$ \\
\hline$S_{14,4}$ & $1.77234 \times 10^{-7}$ \\
\hline$S_{14,5}$ & $-1.76094 \times 10^{-9}$ \\
\hline$S_{14,6}$ & $4.30073 \times 10^{-9}$ \\
\hline &
\end{tabular}

\begin{tabular}{|c|c|}
\hline$S_{14,7}$ & $-2.43475 \times 10^{-10}$ \\
\hline$S_{14,8}$ & $-1.42072 \times 10^{-11}$ \\
\hline$S_{14,9}$ & $4.1348 \times 10^{-13}$ \\
\hline$S_{14,10}$ & $8.33334 \times 10^{-15}$ \\
\hline$S_{14,11}$ & $6.89565 \times 10^{-16}$ \\
\hline$S_{14,12}$ & $-3.88959 \times 10^{-16}$ \\
\hline$S_{14,13}$ & $3.71979 \times 10^{-18}$ \\
\hline$S_{14,14}$ & $2.08219 \times 10^{-17}$ \\
\hline$S_{15,0}$ & 0 \\
\hline$S_{15,1}$ & $-6.5828 \times 10^{-5}$ \\
\hline$S_{15,2}$ & $9.63909 \times 10^{-5}$ \\
\hline$S_{15,3}$ & $9.90187 \times 10^{-7}$ \\
\hline$S_{15,4}$ & $-7.56365 \times 10^{-7}$ \\
\hline$S_{15,5}$ & $-3.05489 \times 10^{-8}$ \\
\hline$S_{15,6}$ & $-2.45565 \times 10^{-10}$ \\
\hline$S_{15,7}$ & $-1.12172 \times 10^{-11}$ \\
\hline$S_{15,8}$ & $2.66204 \times 10^{-12}$ \\
\hline$S_{15,9}$ & $-2.21231 \times 10^{-14}$ \\
\hline$S_{15,10}$ & $-7.67107 \times 10^{-15}$ \\
\hline$S_{15,11}$ & $-2.08224 \times 10^{-15}$ \\
\hline$S_{15,12}$ & $4.21957 \times 10^{-17}$ \\
\hline$S_{15,13}$ & $1.21087 \times 10^{-17}$ \\
\hline$S_{15,14}$ & $-3.91552 \times 10^{-19}$ \\
\hline$S_{15,15}$ & $-4.94421 \times 10^{-19}$ \\
\hline
\end{tabular}

Goldstein, Poole, Safko:'Classical Mechanics', Addison Wesley; 3 edition ISBN13: 978-020165702(2001)

Hofmann-Wellenhof, B., Moritz, H.:'Physical Geodesy', Springer Wien New York (1967)

Lara, M., Scheeres :'Stability bounds for three-dimensional motion close to asteroids', AAS/AIAA Space Flight Mechanics Meeting (2002)

Miller, J. K., Konopliv, A. S., Antreasian,P. G., Bordi, J. J. , Chesley, S., Helfrich, C. E., Owen, W. M., Wang, T. C., Williams, B. G., and Yeomans, D. K. :'Determination of Shape, Gravity, and Rotational State of Asteroid 433 Eros' Jet Propulsion Laboratory, California Institute of Technology, 4800 Oak Grove Drive, Pasadena, California (2001)

Palacián, J.:" 1992, Teoría del Satélite Artificial: Armónicos Teserales y su Relegación Mediante Simplificaciones Algebraicas," Doctoral Dissertation Universidad de Zaragoza, Spain (1992) 
Palacián, J.:'Closed Form normalization of perturbed two-body problems', Cambrige Chaos, Solitons and Fractals (2002)

Segerman, A. M., Coffey, S.L., :An analytical theory for tesseral gravitational harmonics., Celestial Mechanics and Dynamical Astronomy, vol. 76, 139-156, (2000)

San-Juan, J.F., Abad, A., Scheeres, D.J., Lara, M.:"A First Order Analytical Solution for Spacecraft Motion about (433) Eros', AIAA, Astrodynamics Specialist Conference, California (2002)

San-Juan J. F., Abad, A., Lara, M., Scheeres, D. J.,:"First-order analytic solution for Spacecraft about (433) EROS.', Journal of Guidance, Control and Dynamics, vol. 27, No. 2, pp. 290-293, (2004)

Scheeres, D. J. Marzari, F. Tomasella, L. Vanzani, V.,:'ROSETTA mission: satellite orbits around a cometary nucleus', Planetary and Space Science, 46, 649-671, (1998)

Stiefel, E. L.; Scheifele, G.:'Linear and regular celestial mechanics. Perturbed two-body motion, numerical methods, canonical theory.', Die Grundlehren der mathematischen Wissenschaften, Band 174. Springer-Verlag, New YorkHeidelberg,(1971)

Whittaker, E.T.:" A treatise on the analytical dynamics of particles and rigid bodies; with an introduction to the problem of three bodies', Cambrige University Press (1917)

Wigner, E.D.:'Group Theory and it's application to the Quantum Mechanics of Atomic Spectra', New York, Academic Press (1959) 